Yeast

Yeast 2010; 27: 000-000.

Published online in Wiley InterScience

(www.interscience.wiley.com) DOI: 10.1002/yea.I768

Research Article

\title{
There is a steady-state transcriptome in exponentially growing yeast cells
}

\author{
Vicent Pelechano ${ }^{\#}$ and José E. Pérez-Ortín* \\ Departamento de Bioquímica y Biología Molecular, Facultad de Ciencias Biológicas, Universitat de València, C/Dr. Moliner 50, 46I00 \\ Burjassot, Spain
}

\begin{abstract}
*Correspondence to: José E. Pérez-Ortín,

Departamento de Bioquímica y Biología Molecular, Facultad de Ciencias Biológicas, Universitat de València, CIDr. Moliner 50, 46100 Burjassot, Spain. E-mail: jose.e.perez@uv.es

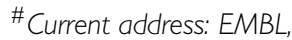
Meyerhofstrasse 1, Heidelberg, Germany.
\end{abstract}

Received: 29 October 2009

Accepted: 10 February 2010

\begin{abstract}
The growth of yeast cells in batches in glucose-based media is a standard condition in most yeast laboratories. Most gene expression experiments are done by taking this condition as a reference. Presumably, cells are in a stable physiological condition that can be easily reproduced in other laboratories. With this assumption, however, it is necessary to consider that the average amount of the mRNAs per cell for most genes does not change during exponential growth. That is to say, there is a steady-state condition for the transcriptome. However, this has not been rigorously demonstrated to date. In this work we take several cell samples during the exponential phase growth to perform a kinetic study using the genomic run-on (GRO) technique, which allows simultaneous measurement of the amount of mRNA and transcription rate variation at the genomic level. We show here that the steady-state condition is fulfilled for almost all the genes during most exponential growth in yeast extract-peptone-dextrose medium (YPD) and, therefore, that simultaneous measures of the transcription rates and the amounts of mRNA can be used for indirect mRNA stability calculations. With this kinetic approach, we were also able to determine the relative influence of the transcription rate and the mRNA stability changes for the mRNA variation for those genes that deviate from the steady state. Copyright $(2010$ John Wiley \& Sons, Ltd.
\end{abstract}

Keywords: transcription rate; steady state; transcriptome; Saccharomyces cerevisiae; mRNA stability

\section{Introduction}

Evaluation of the amount of mRNA (RA) for a given gene by means of northern blot, qPCR or other techniques is a routine in many molecular biology experimental protocols. Comparisons between different physiological situations or between different cell types or mutants are habitually studied. As a significant number of mRNAs can vary their concentrations, even upon subtle changes in internal or external conditions, it is crucial to sample cells in identical situations in order to guarantee repetitiveness and comparativeness between experiments. This is especially true for batch cultures of free-living microorganisms where the growth rate and environment are con- 16 tinuously changing. This problem can be solved by 17 using continuous culture conditions, but such con- 18 ditions are more technically demanding. Therefore, 19 most published experiments use batch conditions. 20 For most batch culture analyses, researchers select 21 exponential growth in a rich medium as the default 22 state for comparison, as it is usually thought to be a 23 physiologically constant condition in which most, 24 or all, mRNAs remain unchanged in a steady-state 25 condition. This can facilitate comparisons between 26 experiments done in different laboratories. How- 27 ever, because the emergence of genomic techniques 28 has raised the possibility of simultaneously quantifying most cellular mRNAs at the same time, the 
1 need for a rigorous demonstration of the steady-

2 state condition now extends to thousands of genes.

3 One of the most studied organisms in func-

4 tional genomics is the yeast Saccharomyces cere-

5 visiae. There are hundreds of experiments on

6 this yeast using cells growing on YPD (yeast

7 extract-peptone-dextrose) complete medium in

8 the 'exponential growth condition'. It is known,

9 however, that many genes vary during exponen-

10 tial growth when cells approach the diauxic shift

11 (DeRisi et al., 1997) or change in a growth rate-

12 dependent manner (Regenberg et al., 2006). As

13 stated above, the use of chemostats has been

14 described as a more reliable way to avoid such vari-

15 ations because they keep culture conditions stable

16 (Daran-Lapujade et al., 2009; Hayes et al., 2002).

17 However, most yeast researchers have used, and

18 still use, the exponential growth condition in batch

19 cultures for their experiments.

20 Although the majority of studies focus only on

21 the determination of RA, it is becoming increas-

22 ingly clear that gene expression should be stud-

23 ied as a kinetic process in which the amount of

24 mRNA is controlled not only by transcription but

25 also by the influence of mRNA stability (RS, also

26 called mRNA half-life; Pérez-Ortín et al., 2007). In

27 exponential growth a dynamic steady state can be

28 assumed for RA, i.e. the transcription and degrada-

29 tion rates (TR and DR, respectively) are equal. TR

30 follows a zero-order kinetics (does not depend on

31 RA), whereas DR follows first-order kinetics:

$$
D R=k_{\mathrm{d}} R A
$$

Therefore, in steady state:

$$
T R=D R=k_{\mathrm{d}} R A
$$

where $k_{\mathrm{d}}$ is the degradation constant, which is inversely related to $\mathrm{RS}\left(k_{\mathrm{d}}=\ln 2 / R S\right)$. Thus, in this situation, $T R$ can be calculated from experimentally determined RA and stability data (Holstege et al., 1998); alternatively, $D R$ can be calculated from RA and TR data (García-Martínez et al., 2004). Both approaches are becoming increasingly popular for TR or DR calculations, although it has not yet been established whether the true steady-state condition for gene expression actually applies (Pérez-Ortín, 2007). A steady state defined for RA does not necessarily imply steady states for TR and DR. In fact, it would be theoretically possible for TR and
DR to change simultaneously in parallel, keeping 52

RA constant.

In this paper we address the topic of assessing 54 the steady-state condition for mRNA amounts in 55 $S$. cerevisiae cells growing in YPD at the expo- 56 nential phase. First we performed an experiment 57 to determine whether the steady state applies for 58 yeast in which we simultaneously measure TR and 59 RA for all the genes. We also studied the kinetic 60 behaviour of any gene that seems to deviate from it. 61 In this way we have demonstrated that the steady- 62 state condition for RA is true for most of the genes 63 studied, but that there are small but significant 64 changes in some groups of genes, which change 65 either their TR or DR as the culture approaches 66 the diauxic shift. Because TR also remains almost 67 invariable, we can say that there is also a steady 68 state for TR and DR. Finally, we reanalysed pre- 69 viously published data (Radonjic et al., 2005) to 70 expand the time window of our study and to deter- 71 mine how long the steady-state condition is main- 72 tained. We found that the RA steady state is ful- 73 filled over a long period within the exponential 74 phase.

Materials and methods

Yeast strain BQS252 (MATa, ura3-52; derived by 81 sporulation from FY1679) was used. Cells were 82 grown in YPD (yeast extract $1 \%$, peptone $2 \%$, and 83 glucose $2 \%$ ) with agitation $(190 \mathrm{rpm})$ at $28^{\circ} \mathrm{C}$ for 84 the repeated sampling of exponentially growing 85 yeast cells. Cell cultures were grown overnight 86 until they reached the desired $\mathrm{OD}_{600}$. Five time 87 points were taken at $0,10,20,30$ and $40 \mathrm{~min} 88$ after the initial sample, which corresponded to 89 $\mathrm{OD}_{600}=0.36\left(8 \times 10^{6}\right.$ cells $\left./ \mathrm{ml}\right), 0.38,0.41,0.4490$ and 0.47 , respectively. Three biological replicates 91 of the whole experiment were done.

\section{Genomic run-on (GRO) 94}

The GRO was done essentially as described in 96 García-Martínez et al. (2004). Briefly, two aliquots 97 of ca. $4 \times 10^{8}$ cells were harvested at each time 98 point. One aliquot was used directly for the GRO 99 protocol, in order to obtain TR data, while the 100 other one was frozen for subsequent total RNA 101 extraction. The cells of the GRO sample were 102 
1 permeabilized with $0.5 \%$ Sarkosyl and incubated 2 for $20 \mathrm{~min}$ on ice. Then the cells were allowed to 3 extend the nascent RNA in the presence of $\left[\alpha-{ }^{33} \mathrm{P}\right]-$ 4 UTP. Finally, the radioactively labelled RNA was 5 extracted and hybridized onto a nylon macroarray 6 (Alberola et al., 2004). After TR determination, the 7 total RNA sample was labelled by cDNA synthesis 8 with random hexamers and hybridized in the same 9 arrays in order to determine the RA.

\section{Image analysis and data normalization}

Images were quantified using Array Vision software, v. 7.0 (Imaging Research Inc.). The signal intensity for each spot was the background subtracted ARM (artifact removed median) density. Only values that were 1.35 times over the corresponding background were taken as valid measurements. The reproducibility of the replicates was checked using Array Stat software (Imaging Research Inc.). We considered the data to be independent and used a minimum number of two valid replicates in order to calculate the mean and standard deviation (SD) values for every gene. Normalization between conditions was done using the global median method.

\section{Data analysis}

We used the differential gene expression analysis program from the GEPAS suite (Montaner et al., 2006) to analyse whether there was any significantly different slope from 0 for the variation of RA during exponential growth (Radonjic et al., 2005) with regard to a continuous independent variable (time). Similar results were obtained when the culture $\mathrm{OD}_{600}$ was used as a continuous independent variable instead of the time (data not shown).

The detailed RA and TR data for exponential growth between $\mathrm{OD}_{600} 0.36$ and 0.47 was calculated as previously described (García-Martínez et al., 2004; for the whole dataset, see Supporting information, Table S1). Briefly, the intensity of the mRNA or GRO hybridization was normalized using a genomic DNA hybridization signal and the U-richness (GRO) or C-richness (mRNA) correction. We computed the slope of RA and TR variations on a $\log _{2}$ scale with regard to time by assuming that the median TR and RA of the gene population remained constant during the experiment (see
Supporting information, Table S2). Gene functional 52 analyses were done by analysing either individual 53 genes slopes (using FuncAssociate; Berriz et al., 54 2003) or gene sets (using Fatiscan from the BABE- 55 LOMICS suite; Al-Shahrour et al., 2006). To anal- 56 yse the changes in RS, we computed a theoreti- 57 cal RA variation dataset for all the genes, using 58 the RA amount data obtained for the first time 59 point and the experimentally obtained TR data 60 for the whole experiment. Using these data, it is 61 possible to calculate a theoretical RA for each 62 point by assuming that the RS calculated the first 63 time remains constant during the whole experiment 64 (Pérez-Ortín et al., 2007). We compared the dif- 65 ferences between this theoretically computed RA 66 and the actual one, and assumed that the differ- 67 ences found would be due to stability changes 68 during the experiment (see Figure 1A). To obtain 69 a more robust RA variation, we performed a lin- 70 ear regression analysis of the actual and theoretical 71 RA data and computed the predicted RA varia- 72 tion for each gene during our experiment, using 73 all the time points. Finally, we computed the per- 74 centage of actual RA variation and the theoretical 75 one (TR-dependent RA variation) for each gene. 76 The difference between the actual and the theoret- 77 ical RA directly gives the stability-dependent RA 78 variation.

Accession numbers

The genomic data are stored in the Valencia 82

The genomic data are stored in the Valencia 83 Yeast (VYdBase; http://vydbase.uv.es/) and GEO 84 databases. The GEO Accession No. for the set of 85 different hybridizations is GSE11521. 86

\section{Results $\quad 88$}

Verification of the steady state for mRNA Verification of the steady state for mRNA 91 on yeast cells using genomic run-on (GRO) 92 analyses

To determine whether the yeast transcriptome was 94 in a steady-state condition during the exponential 95 phase on the YPD medium, we measured the vari- 96 ation of mRNA at five time points during exponen- 97 tial growth. As most of the published experiments 98 start with a very low OD $_{600}$ after inoculation and 99 reach a middle exponential phase of $0.3-0.6$ at 100 $\mathrm{OD}_{600}$, we decided to monitor the mRNA varia- 101 tion under these conditions. We also used our GRO 102 
(A)

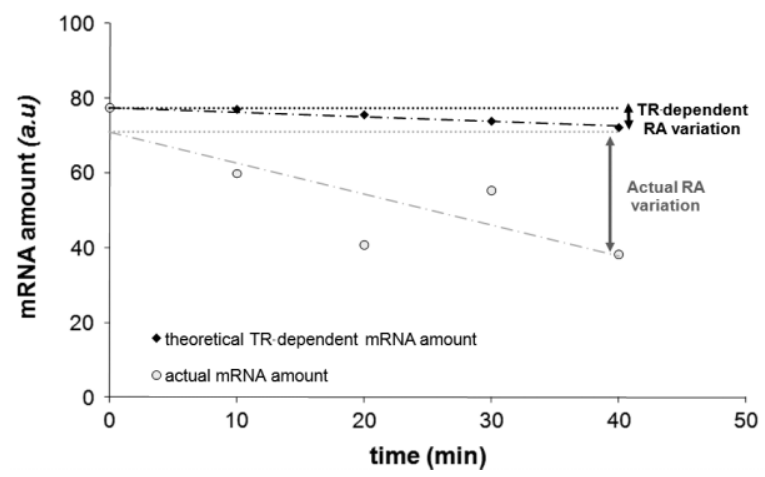

(B)

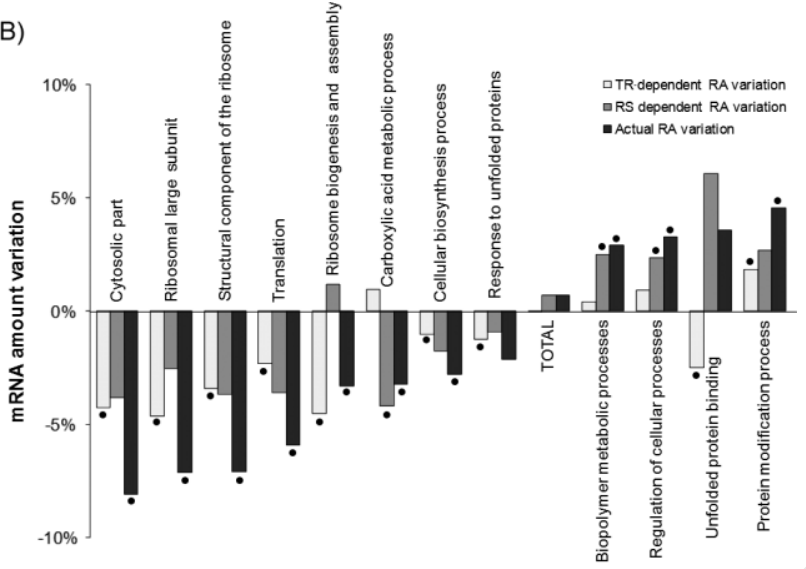

Figure I. Relative contributions of the transcription rate (TR) and mRNA stability (RS) to the mRNA amount (RA) change. (A) Scheme of the protocol used to compute the mRNA variation, which is dependent in either TR or RS. The theoretical RA values in the experiment were computed for each gene by taking in account only the TR variations and by assuming a constant RS (in black). When comparing this theoretical TR-dependent RA change with the actual one (experimentally measured, in grey), it is possible to compute the percentage of RA variation for each gene, which is due to changes in either TR or RS. The data shown correspond to a sample gene (YAL004W) that has been chosen to show an important contribution of RS to RA variation. It is shown in natural scale to make the differences more evident. However its RA slope does not reach the significance cut-off for being statistically different from 0 (in either natural or $\log _{2}$ scale; FDR $p$ value $>0.8$ ). (B) Relative contribution of TR (light grey) or RS (dark grey) to the total RA variation (black) for selected gene groups shown in Table I. Bars represent the median value for the variation for all the genes in each group. The groups with significant differences from 0 (as shown in Tables I and S3) are marked with black dots

1 protocol, which allows the simultaneous determi-

2 nation of TR and RA data. In this way, if the 3 steady-state condition was verified, we could also 4 calculate the mRNA stabilities from the RA and 5 TR data (García-Martínez et al., 2004).

We performed an experiment in triplicate for the five time points at $\mathrm{OD}_{600} 0.36-0.47$ (spanning $40 \mathrm{~min}$, at $10 \mathrm{~min}$ intervals), using cultures inoculated $16 \mathrm{~h}$ beforehand. Under these conditions, the diauxic shift started several hours later at $\mathrm{OD}_{600} \approx 2$ (V. Pelechano, unpublished observation). All 15 samples were subjected to the GRO protocol for calculating RA and TR. The repetitiveness of the samples was very good (see Supporting information, Figure S1). Pearson's correlation was, on average, 0.934 for the TR data and 0.936 for the RA data between the biological replicates. The correlation for the data points for a single growth curve was even better: 0.965 for TR and 0.964 for RA. This suggests that there is more biological variation between growth curves in different experiments than within the time points of a single exponential phase curve. However, as we fused the different replicates, only the time-dependent variations (common for all three experiments) were 26 taken into account.

We reasoned that the slight changes occurring 28 during growth in the culture composition would 29 affect the cells' physiology, leading to a continuous 30 change in RA or TR, which would be visible as 31 positive or negative slopes in their respective plots. 32 In this way, artifactual or random changes would 33 not be accounted for. However, when we analysed 34 these data using the GEPAS suite (a differential 35 expression analysis tool; Montaner et al., 2006), no 36 individual slope of any gene differed significantly 37 from zero $($ FDR $>0.6)$. This result confirms that 38 the steady-state condition applies for all (at least most of) the yeast genes. Therefore, no important error is associated when taking samples for mRNA quantification from cells growing in the early exponential phase. Thus, either TR or RS can be calculated in these growth conditions from the RA experimental data and either from the respective $\mathrm{RS}$ data, measured by conventional methods (Grigull et al., 2004), or from the TR data, measured by GRO by means of mathematical calculations (Pérez-Ortín, 2007). 27

\section{(3)} 33 34 $(35$ 
1 However, the behaviour of genes can be too 2 subtle to be discovered from studying single genes 3 tendencies only. Therefore, in order to analyse 4 this result in more depth, we used a tool from 5 the BABELOMICS suite (Fatiscan algorithm; Al6 Shahrour et al., 2006), which scans the whole set 7 of gene data to discover any common trends in 8 the GO categories. Specifically, this algorithm does 9 a segmentation test that checks whether there are 10 any significant asymmetries in the distribution of 11 each GO. In this way, minor changes that are often 12 too minor to be significant for individual genes 13 can be statistically stressed by the common GO 14 group behaviour. We scanned all the GO categories 15 at all the possible levels. With this analysis, we 16 found that some groups significantly increased 17 or decreased during the experiment (Table 1A). 18 Moreover, as we simultaneously measured RA 19 and TR, we were able to distinguish between the 20 different regulatory strategies.
Some group changes were detected in both TR and RA, but others were observed in only one of them. For instance, the translation-related categories were seen to lower RA and TR, which is in agreement with the decrease noted in the cell duplication rate that the culture would undergo sev- 26 eral hours afterwards. Moreover, the differences 27 between RA and TR behaviours illustrate the possi- 28 bility of detecting the regulatory strategies followed 29 for the mRNA changes: are the changes in RA 30 due to changes in transcription or to changes in 31 mRNA stability? If we assume that the steady- 32 state condition applied for this experiment, then 33 we could calculate the respective influence of TR 34 and RS on the RA change (for a detailed expla- 35 nation, see Figure 1A and Materials and methods). 36 With this protocol, we were able to calculate their 37 relative influence on the changes noted in each 38 GO category, as seen in Table 1 and Figure 1B. 39 We analysed the slope of the RA variation that 40 could be explained by RS changes using Fatiscan. 41 Then we selected the groups with either a sig- 42 nificant positive contribution (meaning increased 43 stability) or a significant negative one (meaning 44 decreased stability) (Table 1B). The fact that the 45 number of statistical significant groups found for 46 the RS changes is smaller than the ones found in 47 the direct measures could be explained mainly by 48 two factors; because it only takes into account the 49 part of the variation for the RA, due to stability 50

Table I. Functional groups with a significant deviation from the steady state during exponential growth

(A) Direct measures

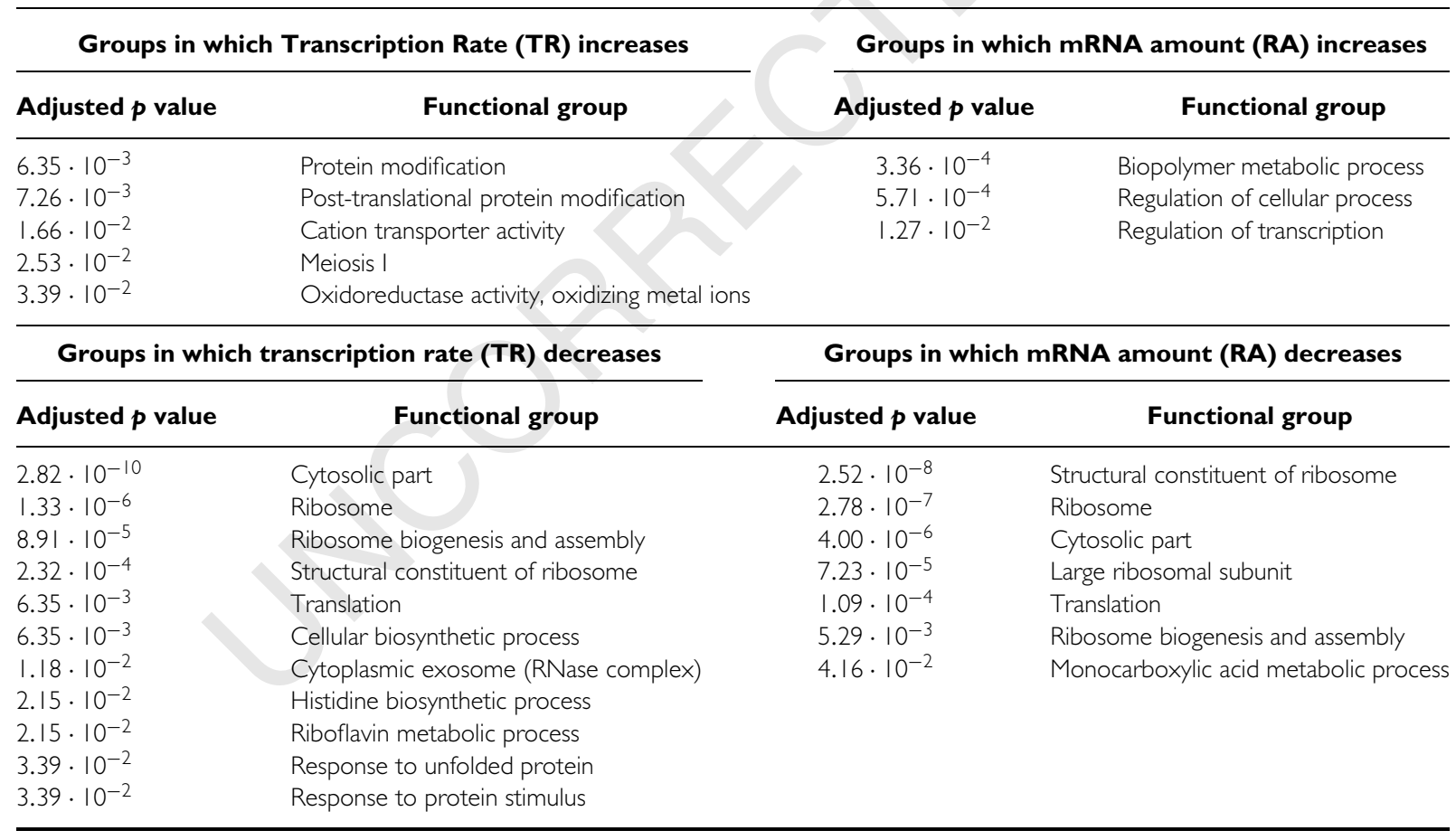


Table I. Continued

(B) Indirect measures

\begin{tabular}{|c|c|c|c|}
\hline \multicolumn{2}{|c|}{ Groups in which mRNA stability (RS) increases } & \multicolumn{2}{|c|}{ Groups in which mRNA stability (RS) decreases } \\
\hline Adjusted $p$ value & Functional group & Adjusted $p$ value & Functional group \\
\hline $1.07 \cdot 10^{-5}$ & Biopolymer metabolic process & $2.06 \cdot 10^{-3}$ & Organic acid metabolic process \\
\hline $2.06 \cdot 10^{-3}$ & Regulation of biological process & $2.06 \cdot 10^{-3}$ & Carboxylic acid metabolic process \\
\hline $2.86 \cdot 10^{-3}$ & Regulation of cellular process & & \\
\hline
\end{tabular}

(A) Functional groups with significant slopes for experimentally determined (direct measures) TR or RA along the five time points analysed during early exponential growth. (B) Functional groups with a significantly high contribution of RS to the RA increase or decrease (mRNA stabilization or destabilization, respectively). The RS-dependent RA slope was mathematically computed (indirect measures), as explained in Figure IA, and used for the functional analysis. Only some representative GO are shown (for the complete list, see Supporting information, Table S3). All the analyses were done using the Fatiscan algorithm from BABELOMICS (Al Shahrour et al., 2006); the FDR-adjusted $p$ values are shown.

1 changes, and because it is an indirect measure.

2 The fact of being an indirect measure depending

3 on the TR and RA data makes it more noisy (in

4 fact the SD for the RS-dependent RA slopes is

5 larger $(0.0074)$ than that from TR or RA direct

6 measures $(0.0058$ and 0.0057 , respectively). It can

7 be seen that both TR and RS cooperate to either

8 lower or increase RA for most categories; that is to

9 say, homodirectional changes occurred, although in

10 variable proportions. In some groups, however, the

11 changes observed operated in opposite senses. RiBi

12 genes had a decreased RA, due to a large effect

13 on TR that compensated slight mRNA stabiliza-

14 tion, whereas unfolded protein response mRNAs

15 increased because of strong mRNA stabilization,

16 despite a certain decrease in TR. It is important to

17 note that all these changes were very subtle, and

18 could be taken in account only for those GOs in

19 which significant changes for the kinetic param-

20 eters had been previously detected. These results

21 illustrate the variable methods used by the yeast

22 cell to change the mRNA level (Pérez-Ortín et al.,

23 2007). experiment, the authors analysed the transcriptome 36 of wild-type yeast (S288c background) growing in 37 a batch culture in YPD since the inoculation on 38 fresh medium to the late stationary phase. They use 39 $\mathrm{OD}_{600}=0.5$ as the starting condition, which pre- 40 cludes a direct comparison with our conditions. The 41 growth curve included nine experimental points in 42 the exponential phase in the range 3.9-9 h after 43 inoculation (see Figure 1A from Radonjic et al., 44 2005). This study measured only the RA changes, 45 but this is enough to check the steady-state condi- 46 tion. First, we analysed this data using the GEPAS 47 suite (a differential expression analysis tool; Mon- 48 taner et al., 2006). Probably because the analysed 49 time window in this experiment is longer, covering 50 more physiological variations, the number of genes 51 with a slope different from zero (FDR $<0.01)$ was 52 higher. We obtained 304 genes showing a posi- 53 tive slope, and 271 showing a negative slope when 54 their $\log _{2}$ RA data were plotted against the time 55 course (Figure 2A). They were enriched in some 56 GO categories. Specifically, the mRNA levels of 57 those categories related with respiration and pro- 58 tein catabolism increased, unlike the mRNA levels 59 of those categories related to ribosome biogenesis, 60 which decreased (Figure 2B). Both results fitted the 61 expected changes in cell metabolism, which would 62 take place when entering the diauxic shift some 63 hours later (DeRisi et al., 1997; Radonjic et al., 64 2005), and they are in agreement with the slight 65 changes detected in our previous experiment. A 66 close inspection of the results, however, showed 67 that most of the changes noted in relation to the 68 initial exponential phase at $3.9 \mathrm{~h}$ in those genes 69 took place at the last two time points analysed, 70 


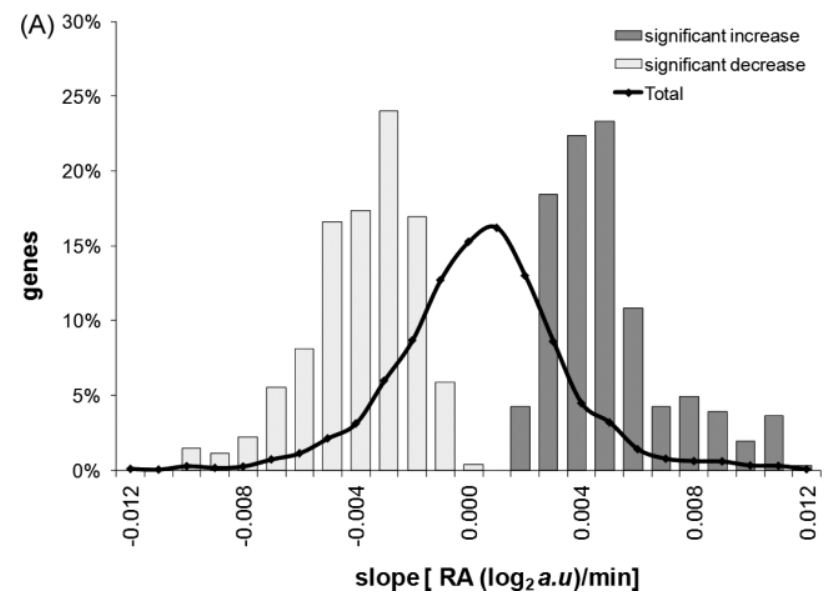

(B) Gene groups with a significant positive RA slope for 3.9-9 $\mathrm{h}$

$p$-value $p$-adj GOAttribute

$3.65 \cdot 10^{-15}<0.001$ vacuolar protein catabolic process

$5.73 \cdot 10^{-11} \quad<0.001 \quad$ response to abiotic stimulus

$1.08 \cdot 10^{-10}<0.001 \quad$ cytoplasmic part

$8.30 \cdot 10^{-10}<0.001$ mitochondrial part

$1.17 \cdot 10^{-07}<0.001 \quad$ oxidation reduction

$3.75 \cdot 10^{-07}<0.001$ actin cortical patch

$1.03 \cdot 10^{-06}<0.001 \quad$ oxidoreductase activity

Gene groups with a significant negative RA slope for $3.9-9 \mathrm{~h}$

$p$-value $\quad p$-adj GoAttribute

$3.15 \cdot 10^{-41}<0.001 \quad$ nucleolus

4.12.10.32 $<0.001$ ribosome biogenesis

$9.20 \cdot 10^{-31}<0.001 \quad$ rRNA processing

$3.91 \cdot 10^{-13}<0.001 \quad$ ribosomal large subunit biogenesis

4.53.10.09 $<0.001$ nucleus

Figure 2. mRNA amount variation during exponential growth. (A) Histogram of slopes for the mRNA amount (RA) variation (in $\log _{2}$ scale for arbitrary units) as a function of time $(\mathrm{min})$ for the $3.9-9 \mathrm{~h}$ interval, according to Radonjic et al. (2005). The distribution of all the gene slopes is shown as a black line. Bars represent the genes with slopes that significantly differ (positively in dark grey and negatively in light grey) from 0 (FDR $<0.0 \mathrm{I}$ ). The $y$ axis represents the percentage of genes, with regard to the total number analysed, belonging to each class. (B) The gene groups that are significantly enriched in genes that increase or decrease RA. Significance was calculated using FuncAssociate (Berriz et al., 2003) Only the most representative GO categories are shown

Table 2. Change in the mRNA amount slope during exponential growth

\begin{tabular}{|c|c|c|c|c|c|c|}
\hline Functional group & $3.9-4.75 h$ & $4-5.25 \mathrm{~h}$ & $4.25-5.75 h$ & $4.75-6.5 h$ & $5.25-7.25 \mathrm{~h}$ & $5.75-9 h$ \\
\hline Cytoplasm & $\uparrow 2.29 \cdot 10^{-8}$ & $\uparrow 9.53 \cdot 10^{-8}$ & $\uparrow 2.76 \cdot 10^{-5}$ & $\uparrow 2.05 \cdot 10^{-7}$ & $\uparrow 3.35 \cdot 10^{-16}$ & $\uparrow 1.14 \cdot 10^{-6}$ \\
\hline Mitochondrial part & $\uparrow 3.42 \cdot 10^{-4}$ & - & - & $\uparrow 3.78 \cdot 10^{-10}$ & $\uparrow 2.88 \cdot 10^{-16}$ & $\uparrow 5.54 \cdot 10^{-12}$ \\
\hline Oxidoreductase activity & $\uparrow 2.31 \cdot 10^{-6}$ & - & & & $\uparrow 4.72 \cdot 10^{-4}$ & $\uparrow 2.03 \cdot 10^{-7}$ \\
\hline Alcohol metabolic process & $\uparrow 6.73 \cdot 10^{-3}$ & - & & & - & $\uparrow 5.55 \cdot 10^{-3}$ \\
\hline $\begin{array}{l}\text { Response to unfolded } \\
\text { protein }\end{array}$ & $\uparrow 4.67 \cdot 10^{-3}$ & & & & $\uparrow 3.07 \cdot 10^{-9}$ & $\uparrow 4.12 \cdot 10^{-3}$ \\
\hline Response to stress & - & - & - & - & $\uparrow 1.56 \cdot 10^{-3}$ & $\uparrow 5.18 \cdot 10^{-3}$ \\
\hline Integral to membrane & $\uparrow 1.34 \cdot 10^{-5}$ & $\uparrow 1.00 \cdot 10$ & $.59 \cdot 10^{-4}$ & $\downarrow 4.44 \cdot 10^{-4}$ & - & - \\
\hline $\begin{array}{l}\text { Ribosome biogenesis and } \\
\text { assembly }\end{array}$ & $\downarrow 7.85 \cdot 10^{-12}$ & 475 & & $\downarrow 1.21 \cdot 10^{-3}$ & $\downarrow 5.56 \cdot 10^{-24}$ & $\downarrow 1.05 \cdot 10^{-28}$ \\
\hline $\begin{array}{l}\text { Structural constituent of } \\
\text { ribosome }\end{array}$ & $\downarrow 6.20 \cdot 10^{-3}$ & & & - & $\downarrow 1.17 \cdot 10^{-17}$ & $\downarrow 8.83 \cdot 10^{-3}$ \\
\hline Translation & $\downarrow 6.09 \cdot 10^{-4}$ & & - & - & $\downarrow 1.59 \cdot 10^{-12}$ & $\downarrow 1.97 \cdot 10^{-6}$ \\
\hline Nucleus & $\downarrow 8.04 \cdot 10^{-14}$ & $\downarrow 2.04 \cdot 10^{-6}$ & - & - & $\downarrow 3.13 \cdot 10^{-8}$ & $\downarrow 1.83 \cdot 10^{-6}$ \\
\hline
\end{tabular}

Gene-set enrichment analysis using Fatiscan (Al Shahrour et al., 2006) according to the RA slopes at different intervals during exponential growth (Radonjic et al., 2005). The categories which increase RA ( $\uparrow$, enriched in the positive slopes) are shown in bold type, and the categories which decrease RA ( $\downarrow$, enriched in the negative slopes) are depicted in normal type. All the slopes were computed using four time points. Only some representative functional groups are shown.

1 i.e. at 7.25 and $9 \mathrm{~h}$ after inoculation. When we 2 considered only the first seven time points, from 33.9 to $6.5 \mathrm{~h}$, no gene's slope significantly differed 4 from zero when we used the same criteria. This 5 result confirms that the time window in which a 6 steady state for the transcriptome can be widely 7 assumed is extended to up a couple of hours before 8 the diauxic shift.
In order to confirm the small deviations from 9 the steady state that we were able to calculate 10 in our previous experiment, we used the same 11 gene set enrichment analysis (Fatiscan algorithm; 12 Al-Shahrour et al., 2006) to detect the groups of 13 the related genes showing slight but significant 14 changes in their RA in the Radonjic et al. (2005) 15 data. Table 2 shows how the RA of some GO 16 
1 categories tended to increase ( $\uparrow$, bold numbers) or

2 decrease $(\downarrow)$ along the growth curve. As expected,

3 the respiration and stress response categories were

4 significantly represented in the RA increase, while

5 the translation categories were significantly repre-

6 sented in the RA decrease.

7 Therefore, although we conducted a kinetically

8 detailed study of the transcriptome only during

9 mid-exponential growth, these results confirm that

10 our conclusions can be extrapolated to most of the

11 exponential growth phase.

\section{Discussion}

It is commonly assumed that mRNAs are approximately in a steady state during exponential growth in a free-living microorganism. For instance, we have used this assumption in order to calculate the mRNA stabilities in the yeast $S$. cerevisiae by means of GRO experiments (García-Martínez et al., 2004). However, an experimental demonstration is currently lacking.

Here we report a detailed study of the gene expression in yeast during exponential growth. In this experiment we simultaneously checked both RAs and TRs. The general conclusion is that a steady state for the transcriptome and transcription rates can be assumed, and that small deviations from it can be detected, but only when looking at all the genes from a given GO at the same time. This last analysis procedure is much more sensitive because it detects common tendencies for a group of related genes. However, when we analysed them individually, each yeast gene was within the steady-state condition for its mRNA. In addition, our kinetically orientated approach enables us to determine the respective contributions of TR and RS to the putative changes in RA. For most cases, both changes work in the same direction. However, certain exceptions indicate how the cell uses mRNA stability as an additional controller of the gene expression. Moreover, the demonstration of steady state for TR allows the conclusion that the cells keep the RA values constant by also maintaining constant turnover, not by coordinately changing TR and DR - a more complicated option but theoretically possible.

To extend our results and to determine how long the steady state for the transcriptome can be assumed during exponential growth, we used

data from a comprehensive microarray study by 52 Radonjic et al. (2005), which is representative for 53 many others. Using these data, we verified that the 54 steady-state condition is met by a sample series 55 over a long period (for at least $3 \mathrm{~h}$ and up to $2 \mathrm{~h} 56$ before the diauxic shift). There is nevertheless a 57 significant tendency of the mRNAs related with 58 the growth rate (translation) to decrease and the 59 genes related to the diauxic shift (mitochondria) 60 to increase. This probably reflects the cell's early 61 response to the forthcoming changes to take place 62 in metabolism, and corroborates the suggestion that 63 yeast cells use a feed-forward strategy, anticipating 64 growth changes with gene expression changes 65 (Levy and Barkai, 2009).

Having established that the steady-state condi- 67 tion during exponential growth had been fulfilled, 68 we can say that our protocol to calculate mRNA 69 half-lives indirectly, as well as that used by other 70 authors to calculate TR from the RA and RS data, 71 have been verified. The correlations for direct and 72 indirect computed TRs are about 0.5 (Pérez-Ortín, 73 2007). This is a significant correlation but is not as 74 high as expected. There may be several reasons for 75 this: first, the mathematical error associated with 76 indirect calculations; second, the error associated 77 with the direct measurement of the mRNA half- 78 life (see below). Both problems are unavoidable 79 and will mostly introduce random noise into the 80 indirect data and, therefore, decreased correlation. 81 Another reason for the low correlation obtained 82 could be that indirect TR measures the appear- 83 ance of mature mRNA in the cytoplasm, whereas 84 GRO (or other methods to estimate the TR, such 85 as RPCC (Pelechano et al., 2009), measures the 86 density of RNA polymerases. Density can only be 87 converted into TR by assuming a constant RNA 88 pol $I_{A}$ speed (Hirayoshi and Lis, 1999). Direct 89 methods measure 'nascent TR', which can differ 90 from 'mature TR' (increase of mature, cytoplasmic 91 mRNAs over time) if the proportion of produc- 92 tive transcription (finished mRNAs) is not the same 93 for all the genes, or if the transport of mRNAs is 94 not equally efficient for them all. This discrepancy, 95 however, is potentially interesting because it opens 96 a way to determine the differences in transcription 97 elongation or mRNA processing between different 98 groups of genes.

The calculation of the RS data indirectly from 100 the RA and TR data can also be compared with 101 those calculated by direct methods. In this case, 102 
no correlation exists (see Pérez-Ortín, 2007). This result is surprising and suggests potential confusing influences when mathematically operating with variables that have already been correlated. For instance, TR and RA correlate positively, and both correlate negatively with RS (García-Martínez et al., 2007). Thus, when computing TR with the RA and RS data (TR $\alpha_{\lambda}$ RA/RS), we obtain a new dataset by dividing one dataset that correlates positively with the TR by one that is inversely correlated. The result is, therefore, a new dataset that should mathematically correlate positively with the TR, as is in fact the case. However, when we attempted to compute RS using the TR and RA data (RS $\left.\alpha_{\lambda} \mathrm{RA} / \mathrm{TR}\right)$, we divided two datasets that negatively correlated to RS. Thus, this negative correlation in the new dataset decreased, due to a confusing effect of the different variables. Another source of discrepancy is the dilution effect caused by the continuous growth of the culture when calculating indirect RS that contributes to mRNA concentration reduction besides the mRNA degradation itself (Alon, 2006), whereas it does not affect indirect methods because the growth of the culture is stopped due to the transcription stop. Additional limitations in the calculations are related to the use of nascent TR (see above) and the well-known problems brought about by the stressing situation caused to cells because of the transcription stop that they require (Grigull et al., 2004; Pérez-Ortín et al., 2007).

Finally, the confirmation of a transcriptional steady state during the exponential phase means that the functional analyses of gene expression done in yeast to date are reliable, as this steady state confirms that the different time points within that phase can be considered as identical with regard to all mRNA levels.

\section{Acknowledgements}

We wish to thank Dr S. Chávez and Albert Sorribas for critically reading the manuscript, and all the members of the Yeast Functional Genomics Group for discussions. We are especially grateful to José García-Martínez (DNA-chip service at the SCSIE) for performing the DNA macroarrays. V.P. was partially funded by a FPI fellowship from the Generalitat Valenciana (Valencian Regional Government). This work was supported by Grant No. BFU2007-67575-

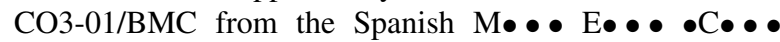
and Grant No. ACOMP/2009/368 from the Generalitat Valenciana (to J.E.P.-O.).

\section{Supporting information on the internet}

The following suporting information may be found 54 in the online version of this article: 55

Table S1. TR and RA values for the experiment 56

Table S2. Relative contribution of TR to RA 57 changes

Table S3. Complete lists of functional groups 59 with a significant deviation from the steady state 60 during exponential growth

Figure S1. Correlation between the different time points for the amount of mRNA (RA) and the transcription rate $(\mathrm{TR})$

\section{References}

Al-Shahrour F, Minguez P, Tarraga J, et al 2 LOMICS: a systems biology perspective in the functional anno- 70 tation of genome-scale experiments. Nucleic Acids Res 34: 71 W472-476.

Alberola TM, García-Martínez J, Antúnez O, et al. 2004. A new set of DNA macrochips for the yeast Saccharomyces cerevisiae: features and uses. Int Microbiol 7: 199-206.

Berriz GF, King OD, Bryant B, et al. 2003. Characterizing gene sets with FuncAssociate. Bioinformatics 19: 2502-2504.

Daran-Lapujade P, Daran JM, van Maris AJ, et al. 2009. Chemostat-based micro-array analysis in baker's yeast. $A d v$ Microb Physiol 54: 257-311.

DeRisi JL, Iyer VR, Brown PO. 1997. Exploring the metabolic and genetic control of gene expression on a genomic scale. Science 278: 680-686.

García-Martínez J, Aranda A, Pérez-Ortín JE. 2004. Genomic 82 run-on evaluates transcription rates for all yeast genes and 83 identifies gene regulatory mechanisms. Mol Cell 15: 303-313.

García-Martínez J, González-Candelas F, Pérez-Ortín JE. 2007. Common gene expression strategies revealed by genome-wide analysis in yeast. Genome Biol 8: R222.

Grigull J, Mnaimneh S, Pootoolal J, et al. 2004. Genome-wide 87 analysis of mRNA stability using transcription inhibitors and 88 microarrays reveals posttranscriptional control of ribosome 89 biogenesis factors. Mol Cell Biol 24: 5534-5547.

Hayes A, Zhang N, Wu J, et al. 2002. Hybridization array 90 technology coupled with chemostat culture: tools to interrogate 91 gene expression in Saccharomyces cerevisiae. Methods 26: 92 281-290.

Hirayoshi K, Lis JT. 1999. Nuclear run-on assays: assessing transcription by measuring density of engaged RNA polymerases. Methods Enzymol 304: 351-362.

Holstege FC, Jennings EG, Wyrick JJ, et al. 1998. Dissecting the 96 regulatory circuitry of a eukaryotic genome. Cell 95: 717-728. 97

Levy S, Barkai N. 2009. Coordination of gene expression with 98 growth rate: a feedback or a feed-forward strategy? FEBS Lett 99 583: $3974-3978$.

Montaner D, Tarraga J, Huerta-Cepas J, et al. 2006. Next station 100 in microarray data analysis: GEPAS. Nucleic Acids Res 34: 101 W486-491. 
Pelechano V, Jimeno-González S, Rodriguez Gił A, et al. 2009. Regulon-specific control of transcription elongation across the yeast genome. PLoS Genet 5: e1000614.

Pérez-Ortín JE. 2007. Genomics of mRNA turnover. Brief Funct Genom Proteom 6: 282-291.

Pérez-Ortín JE, Alepuz PM, Moreno J. 2007. Genomics and gene transcription kinetics in yeast. Trends Genet 23: 250-257.

Radonjic M, Andrau JC, Lijnzaad P, et al. 2005. Genome-wide analyses reveal RNA polymerase II located upstream of genes

poised for rapid response upon S. cerevisiae stationary phase 31 exit. Mol Cell 18: 171-183. 32

Regenberg B, Grotkjaer T, Winther O, et al. 2006. Growth-rate 33 regulated genes have profound impact on interpretation of 33 transcriptome profiling in Saccharomyces cerevisiae. Genome 34 Biol 7: R107.

Alon U. 2006. An Introduction to Systems Biology: Design 36 Principles of Biological Circuits. Chapman \& Hall/CRC Mathematical \& Computational $\bullet$ Biology: 
IMPORTANT NOTE: Please mark your corrections and answers to these queries directly onto the 54 proof at the relevant place. Do NOT mark your corrections on this query sheet.

\section{Queries from the Copyeditor:}

AQ1 Please spell out MEC in full

AQ2 Insert CITY of publisher

$\equiv$\begin{tabular}{ll}
\hline & 59 \\
& 60
\end{tabular} 